\section{„Tipps und Tricks“ für schwierige Gespräche mit Patientinnen}

Patientenzentrierte Kommunikation ist nichts für Zartbesaitete: die enormen Herausforderungen an professionelle Kommunikation, mit denen es Ärzte in der Gynäkologie zu tun haben Situationen, in denen sie sich rat-und hilflos erleben, in der Zwickmühle, ohne Möglichkeit der Rücksprache im Nachtdienst, werden nicht nur von manchen Vorgesetzten verharmlost oder übersehen. Genauso selbstverständlich und unterbewertet werden die vielen Beispiele gelungener, hilfreicher Kommunikation, mit denen Ärzte den Patientinnen zur Seite stehen und sie nach Kräften unterstützen.

So sehr man es sich wünschen würde: eine Wunderkiste mit den in jeder Situation passenden „richtigen“ Worten oder Verhaltensweisen gibt es nicht. Was einer Patientin dabei hilft, ihrem Arzt zu vertrauen, ist für eine andere Patientin völlig ungeeignet. Auch zwischen (Kommunikations-)Experten und Patienten gehen die Auffassungen über „gute“ Kommunikation gewaltig auseinander; ihnen geht es oft um ganz andere Aspekte in der Interaktion, als von Ärzten vermutet. Vermeintlich falsch gewählte Begriffe hat die Patientin gar nicht gehört, weil sie sich bei dieser Ärztin gut aufgehoben fühlt; während ihr unbedacht geäußerte Worte oder unerbetene Informationen - „sollten wir Metastasen ausschließen“ - fortwährend im Kopf kreisen und im weiteren Gespräch ihre Aufmerksamkeit absorbieren.

\section{Patientenzentrierte Kommunikati-} on: Haltung und „Technik $\nabla$

Was die meisten Patientinnen intuitiv sehr wohl wahrnehmen: ob ihr ärztliches Gegenüber an ihnen interessiert, um Verstehen und so etwas wie Aufrichtigkeit bemüht ist, und ob sie sich als Person wahrgenommen fühlen. Dazu gehört, dass die Patientin den Arzt mit seiner unverwechselbaren persönlichen Note als „echt“ wahrnimmt, wobei Form und Inhalt übereinstimmen. Angelernte stereotype Äußerungen, etwa der berühmte „Warnschuss“ vor schlechten Nachrichten
- „Es tut mir leid, dass ich keine guten Nachrichten für Sie habe.“ - werden von Patientinnen leicht als unpersönlich, oder aufgesetzt erlebt und irritieren eher als Vertrauen zu schaffen.

Häufig versuchen Ärzte, vorauszuahnen, wie eine Patientin z.B. auf eine schlechte Nachricht reagieren wird - sie „zerbrechen sich den Kopf der Patientin“. Ärzte sind keine Hellseher - sie müssen und können nicht wissen, was einer Patientin durch den Kopf geht; aber sie können versuchen, es mit ihr gemeinsam herauszufinden: mit einer Einstellung, die sich als engagiertes Interesse am persönlichen Erleben der Patientin bezeichnen lässt. Als „Technik“ hierfür eignen sich offene Fragen - etwa nach den Gedanken und Gefühlen, die diese Patientin gerade beschäftigen, nach den Fragen, die ihr durch den Kopf gehen - und keine vorgefertigten, vermeintlich tröstlichen Antworten - „Sie werden sehen, so schlimm ist es gar nicht.“ oder „Sie brauchen sich keine Sorgen zu machen."- mit denen man meistens haarscharf daneben liegt. Oft genug mit der Folge, dass sich die Patientin unverstanden oder allein gelassen fühlt. Mit offenen Fragen - „Wie ist es Ihnen seit der letzten Chemotherapie ergangen?“ „Was sind Ihre Bedenken gegenüber der antihormonellen Therapie?" - vermittelt man nicht nur das Interesse am subjektiven Erleben der Patientin; meistens kommt man schneller zum Kern eines Problems, erfährt eher von körperlichen und seelischen Beschwer- den, die Patientinnen auf geschlossene Fragen - „War Ihnen übel nach der letzten Chemotherapie?" - nicht ohne weiteres äußern. Offene Fragen eines engagierten Arztes helfen nicht nur, Gesprächszeit zu sparen, sie tragen nachweislich dazu bei, die Compliance - z. B. antihormonelle Therapie bei Brustkrebs-Patientinnen - und auch die Verträglichkeit einer Behandlung zu verbessern.

Es geht also weniger um kommunikative „Skills“, als um das Gestalten einer professionellen Beziehung die von einer Patientin als hilfreich erfahren wird - wobei die Situation im Kreißsaal eine andere ist als in der Brustsprechstunde, in der Praxis eine andere als in der ChemotherapieEinheit oder im OP.

Nirgendwo liegen Leben und Tod oft so nah beieinander wie in der Gynäkologie. Gerade in existenziellen Krisensituationen geht es darum, die besondere Verletzlichkeit der Patientin im Blick zu behalten - die nach außen hin kaum zu erkennen, oft genug hinter einer gefassten Haltung verborgen oder als schwierige Frage geäußert wird (s.u.). Nicht weniger geht es darum, die eigene emotionale Beteiligung als Ärztin, als Arzt anzuerkennen. Es gehört zum ärztlichen Beruf, dass uns das Schicksal von Patientinnen berührt und buchstäblich „unter die Haut“ geht; deshalb geht es immer wieder um eine halbwegs gelingende Balance zwischen Em-

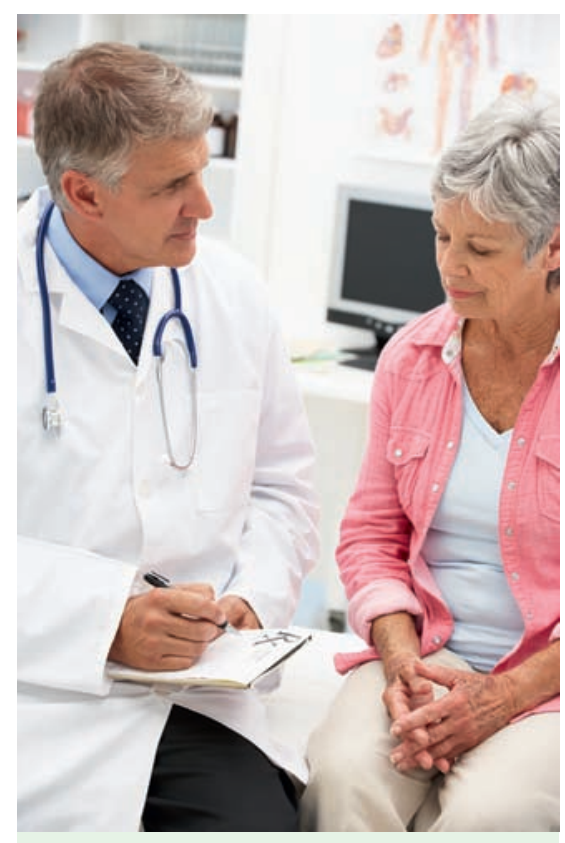

Für Patientinnen ist es wichtig, sich vom Arzt als Person wahrgenommen zu fühlen (Symbolbild, Quelle: shutterstock). 
pathie (nicht: Mitleid!), innerer Distanzierung und Selbstschutz, und um den Erhalt ärztlicher Handlungsfähigkeit. Das gelingt besser, wenn Ihnen eine Patientin oder ein Paar besonders sympathisch ist, die Ihnen spürbar vorbehaltloses Vertrauen entgegen bringen; es gelingt weniger gut, wenn Sie unter Zeitdruck, müde vom Nachtdienst oder nach einer unerfreulichen Auseinandersetzung mit Ihrer Oberärztin, einer mürrischen, leicht vorwurfsvollen Frau mitzuteilen haben, dass die Chemotherapie wegen zu niedriger Leukozyten verschoben werden muss. In dieser Situation fällt es zwangsläufig schwerer, sich kurz in die Patientin hinein zu versetzen, und sich ihre nachvollziehbare Enttäuschung vorzustellen, dass sie „umsonst“ so lange gewartet hat, und sich womöglich eigene Pläne zerschlagen haben. Professionelle Kommunikation heißt, sich die momentan begrenzte eigene Empathiefähigkeit zuzugestehen und zu „erlauben“; mit dem Bedauern darüber, dass man der Patientin nur unzureichend gerecht wird; ohne als Folge eines uneingestandenen schlechten Gewissens in eine Rechtfertigungshaltung zu geraten, die keinem nutzt.

Auch wenn es nur wenige allgemein gültige Regeln für gelingende Gespräche, für patientenzentrierte Kommunikation gibt, haben sich einige Anregungen bewährt, die es nicht nur Patientinnen erleichtern, Vertrauen zu fassen. Auch Ärzte machen die Erfahrung, dass ihnen damit Gespräche leichter fallen, und dass es seltener zu Missverständnissen, und zu ,schwierigen Gesprächssituationen' kommt.

\section{Orientierung und Struktur \\ $\nabla$}

Strukturieren Sie möglichst jedes Gespräch: Es erleichtert Ihnen und der Patientin die Orientierung, wenn Sie den zeitlichen Rahmen von vorne herein festlegen,

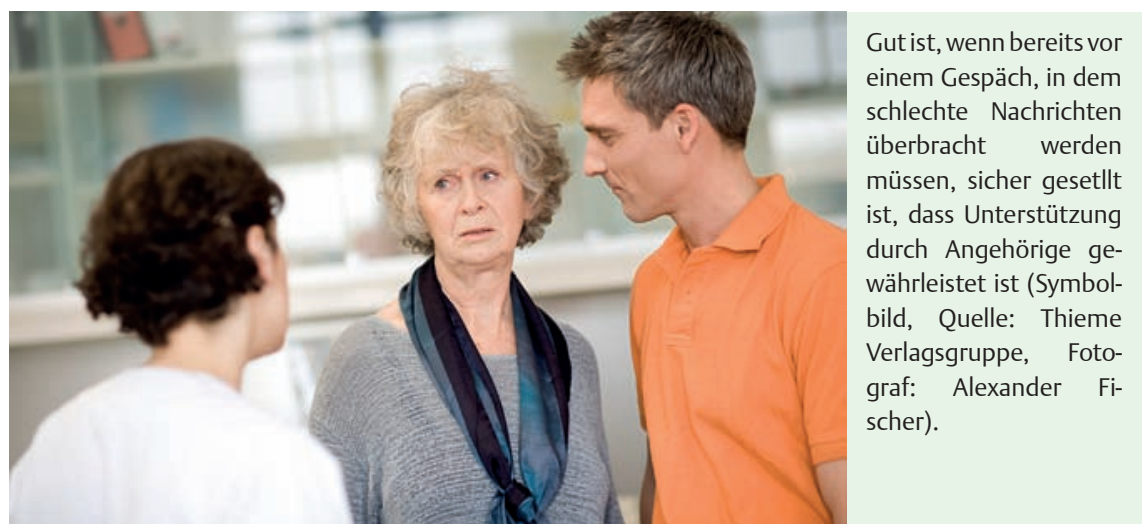

ohne Bedauern wenn es „nur“ 10 Minuten sind; ein konzentriertes 10-minütiges Gespräch kann von Patientin und Ärztin gleichermaßen als ergiebig und ausreichend erlebt werden.

So banal es klingen mag: einigen Sie sich anfangs gemeinsam auf eine Agenda, auf das wichtigste Thema bzw. auf einen Auftrag. Dies beugt Missverständnissen vor und ermöglicht gerade einer ängstlichen oder verunsicherten Patientin, sich mental zu orientieren; z. B. mit der offenen Frage „Was ist Ihnen heute wichtig?“. Damit erfährt man eher von Befürchtungen, z. B. bei einer Routine-Untersuchung in der Schwangerenvorsorge, oder der Nachsorge nach einer Brustkrebs-Erkrankung, die sonst hinter wiederholten, nicht selten zeitraubenden Fragen verborgen, auf Nebenschauplätzen geäußert werden.

Es fördert die Orientierung, wenn Sie Themenwechsel, von Ihnen oder der Patientin initiiert, ankündigen und benennen. Beenden Sie möglichst jedes Gespräch so, dass beiden klar ist, wie es weiter geht, und welche weitere Unterstützung benötigt wird.

\section{Schlechte Nachrichten überbringen \\ $\nabla$}

Welche Situationen, welche Gespräche fallen Ärzten - nicht nur in der Gynäkologie - besonders schwer? Für die meisten ist es, schlechte Nachrichten überbringen zu müssen:

- einer jungen Mutter mitteilen, dass ihr Kind tot geboren wurde

- einem Paar mit unerfülltem Kinderwunsch vermitteln, dass die Entfernung des Uterus aufgrund eines Endometrium-Ca unumgänglich ist

- einer alleinerziehenden Mutter erklären, dass unter Chemotherapie Leber-
Auch langjährige klinische Erfahrung ändert wenig daran, dass einem solche Gespräche nahe gehen; selbst unter erfahrenen Klinikern sind 2 Drittel unsicher oder unzufrieden mit ihren Fähigkeiten, wenn es um den Umgang mit solchen schicksalhaften Mitteilungen geht.

Es lohnt sich, wenn Sie sich vor einem schwierigen Gespräch, bei dem es um das Mitteilen von ,schlechten Nachrichten' geht, ein paar Minuten Zeit nehmen: werden Sie sich Ihrer eigenen Verfassung bewusst, Ihrer Stimmung, den speziellen Erwartungen und vielleicht Befürchtungen vor diesem Gespräch, mit dieser Patientin; machen Sie sich auch Ihre Stärken und Fähigkeiten bewusst; tauschen Sie sich evt. mit Pflegenden oder Kollegen aus: wie nehmen sie die Verfassung der Patientin wahr, gibt es Hinweise für eine besondere Vulnerabilität?

\section{Machen Sie sich klar, dass}

- auch die beste und einfühlsamste Gesprächsführung aus einer schlechten keine gute Nachricht macht,

- Sie nicht verantwortlich, und schon gar nicht „schuld“ daran sind, was Sie zu vermitteln haben,

- Sie aber verantwortlich dafür sind, wie Sie es tun.

Es hat sich bewährt, wenn man sich an einer inhaltlichen Abfolge wie an einem Leitfaden (z.B. anhand des SPIKES-Modells) orientiert. Dazu gehört, dass Sie:

- zunächst basale Informationen - Vorwissen, Vermutungen und Erwartungen - von der Patientin brauchen, die Sie am besten mit offenen Fragen eruieren. An diese Vermutungen anzuknüpfen erleichtert das Übermitteln von schlechten Nachrichten.

- die schlechte Nachricht klar, knapp und verständlich vermitteln, möglichst ohne medizinische Details. Damit berücksichtigen Sie, dass Konzentration und Aufnahmefähigkeit in Ausnahmesituationen zumeist eingeschränkt sind. 
- Pausen von mindestens 3 Sekunden entstehen lassen, während der Sie Blickkontakt halten, sodass sich die Patientin nicht verloren fühlt. Damit lassen Sie ihr Raum für Fragen und können sich einen Eindruck von ihrer Reaktion machen.

- sich mit der Patientin verständigen, welche weiteren Informationen sie jetzt braucht und verarbeiten kann. Verzichten Sie darauf, Informationen (z.B. zur Prognose) zu geben, nach denen die Patientin nicht gefragt hat.

- sich darüber verständigen, wie die Nachricht angekommen ist - „Checking understanding“, evtl. in Folgegesprächen. Gesagt ist keineswegs immer gleichbedeutend mit angekommen; in Ausnahmesituationen sind Fehlwahrnehmungen und Verzerrungen, NichtWahrhaben-Wollen vorübergehend adaptiv und verändern sich - als Ausdruck der seelischen Verarbeitung zumeist innerhalb kurzer Zeit.

- Ihr Mitgefühl - z. B. Bedauern, Enttäuschung, Traurigkeit, Zorn - ggf. und nur wenn authentisch - zum Ausdruck bringen.

- seelische Reaktionen - Weinen, Verzweiflung, Schock, Versteinerung wahrnehmen und anerkennen (z. B. „das ist jetzt ziemlich hart “, „das ist schwer zu verkraften“) und in Kontakt mit der Patientin bleiben, ohne etwas zu ,tun', auch nicht durch vorschnelles Trösten unterbrechen. „Spiegeln“, wenn keine seelische Reaktion erkennbar wird, z. B. „Ich habe den Eindruck, dass Sie das sehr gefasst aufnehmen?".

- das weitere Vorgehen besprechen und planen; es ermöglicht der Patientin eine Perspektive bzw. eine Vorstellung, „dass es weiter geht“.

- sicherstellen, dass soziale Unterstützung durch Bezugspersonen verfügbar ist, ggf. initiieren.

- Ihre weitere Verfügbarkeit - wann immer möglich - anbieten; möglichst Folgekontakt vereinbaren.

Aller Erfahrung nach ist ein so strukturierter Ablauf für Aufklärungsgespräche nicht zeitaufwändig, sondern zeitsparend. Er dient auch der eigenen Orientierung. Den Ablauf sollte man passend zum persönlichen Gesprächsstil und an die jeweilige klinische Situation angepasst, flexibel handhaben. Ob es ein „gutes“ Gespräch war, merkt man am ehesten daran, ob man sich hinterher besser, weniger angespannt oder bedrückt, fühlt als vorher. Patientinnen erinnern auch schwere Auf-

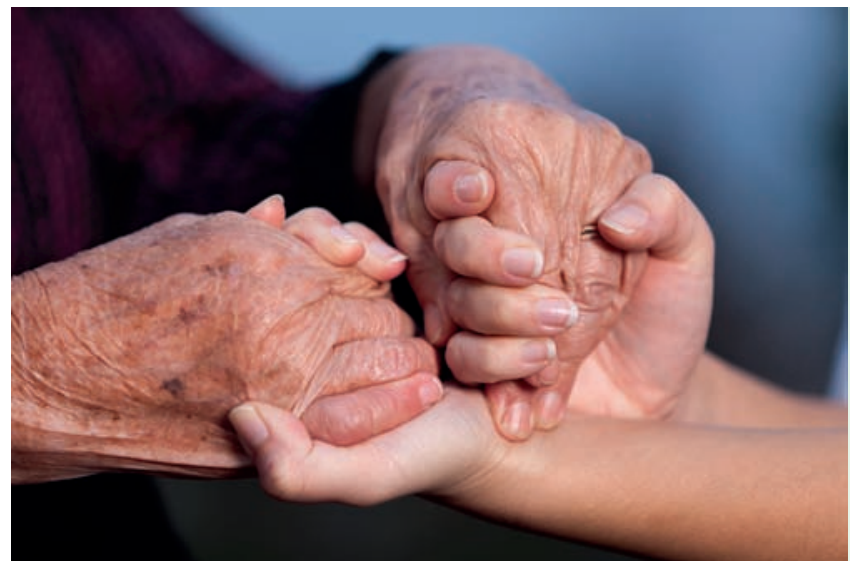

Selbst schwere Aufklärungsgespräche können von Patienten als gut empfunden werden, wenn sie sich vom medizinischen Gesprächspartner respektiert, ermutigt und unterstützt fühlen (Symbolbild, Quelle: istockphoto, Fotograf: Slobodan).

klärungsgespräche als unerwartet wohltuend, wenn sie sich mit ihrem persönlichen Erleben wahrgenommen, verstanden und respektiert, und von ihrem Arzt ermutigt und unterstützt fühlen, auch wenn sich ihr Leben mit der schlechten Nachricht schlagartig verändert hat (,...komisch, ich war hinterher ganz ruhig“).

\section{Mit schwierigen Fragen umgehen}

$\nabla$

„Aber ich werde doch wieder gesund?“

„Sagen Sie mir wie viel Zeit ich noch habe.“

„Dann war also alles umsonst?“

„Können Sie mir denn gar nicht mehr helfen?"

„Dann geben Sie mich also auf?“

„Sie können mich doch nicht einfach sterben lassen!“

... Ob und welche dieser Fragen oder Äußerungen als besonders „schwierig“ erlebt werden, unterscheidet sich zwischen Ärzten, je nach persönlichen Stärken und lebensgeschichtlichen Prägungen. Eine „schwierige“ Frage oder Behauptung merkt man vor allem an der eigenen Reaktion; an mehr oder weniger deutlichen, meist unangenehmen seelischen und körperlichen Sensationen: z.B. das Gefühl von „einer Pistole auf der Brust“, es „verschlägt einem die Sprache“ oder man ertappt sich bei Fluchtimpulsen. Jede dieser Sensationen, so diskret sie auch sein mag, hat vor allem Signalfunktion: sie verweist auf den Appell, den die Patientin in seelischem Ausnahmezustand - Angst, Hilflosigkeit, Verzweiflung - aussendet, um den Arzt zu erreichen, ohne dass dies bewusst, geplant oder beabsichtigt ist; ein Not- und Hilferuf, weil sie sich anders nicht mitteilen kann. Das wichtigste für die Patientin ist, dass der Arzt den Hilferuf hinter der vermeintlichen Sachfrage nicht überhört und dem Impuls widersteht, unmittelbar auf der Sachebene - z. B. mit einer Aussage zur verbleibenden Lebenszeit - zu antworten. Vor jeder Antwort gilt es, dass man sich eine Vorstellung von der Notsituation macht, in der sich die Patientin befindet: z.B. indem man mit offenen Fragen, einer Einladung, zu erzählen, besser verstehen kann, was die Patientin so umtreibt. Auf der Beziehungsebene erfährt die Patientin damit, dass sie ihr Gegenüber, hier den Arzt, erreicht, dass sie mit Hilflosigkeit, Ängsten und Verzweiflung gesehen, und nicht im Stich gelassen wird, dass sie in Ansätzen Sicherheit zurück gewinnt und sich in einer scheinbar aussichtslosen Situation unterstützt weiß.

An dieser Stelle äußern Ärzte nicht selten Widerspruch: Der Verzicht auf eine aufrichtige Antwort sei ein Ausweichen, mit dem das Recht von Patienten, von ihrer begrenzten Lebenszeit zu erfahren ignoriert, und die Gelegenheit, Unerledigtes zu ordnen, vorenthalten würde. Patienten äußern dagegen öfters, dass sie in dieser Situation gar nicht mit einer Antwort auf der Sachebene gerechnet hatten. Manche Patienten wünschen sich im Nachhinein, sie hätten diese Frage nach der verbleibenden Lebenszeit nicht gestellt. Ärztliche Verantwortung und Respektieren der Autonomie eines Patienten schließen sich gegenseitig nicht aus und ergänzen sich bestenfalls, wenn im Gespräch geklärt werden kann, welche Anliegen, welche Themen für die Patientin jetzt wichtig sind, und welche Fragen eine Antwort auf der Sachebene (s. Überbringen schlechter Nachrichten) erfordern.

PD Dr. med. Monika Keller, Sektion Psychoonkologie, Klinik für Allg. Innere Medizin und Psychosomatik, Medizinische Universitätsklinik, Heidelberg, monika.keller@med.uni-heidelberg.de 